\title{
A numerical approximation of the thermal coupling of fluids and solids
}

\author{
Javier Principe $^{* \dagger}$ and Ramon Codina \\ CIMNE, Universitat Politècnica de Catalunya, Jordi Girona 1-3, Edifici C1, 08034 Barcelona, Spain
}

\begin{abstract}
SUMMARY
In this article we analyze the problem of the thermal coupling of fluids and solids through a common interface. We state the global thermal problem in the whole domain, including the fluid part and the solid part. This global thermal problem presents discontinuous physical properties that depend on the solution of auxiliary problems on each part of the domain (a fluid flow problem and a solid state problem). We present a domain decomposition strategy to iteratively solve problems posed in both subdomains and discuss some implementation aspects of the algorithm. This domain decomposition framework is also used to revisit the use of wall function approaches used in this context. Copyright (C) 2008 John Wiley \& Sons, Ltd.
\end{abstract}

Received 6 September 2007; Revised 1 February 2008; Accepted 4 May 2008

KEY WORDS: thermal coupling; thermal boundary layers; domain decomposition; stabilized finite elements

\section{INTRODUCTION}

The problem we analyze in this article is that of the thermal coupling of fluids and solids. This problem is found in any engineering design in which a fluid is used to extract heat from a solid (refrigeration, ventilation, etc.). In fact, many experimental correlations are available [1] in the form of convection coefficients. The objective of this article is to present a domain decomposition approach that permits the separated treatment of a problem in the solid domain and of a problem in the fluid one. Let us emphasize that it is not our intention to use a domain decomposition strategy to perform parallel computations but to treat problems with different physics separately. Moreover, this domain decomposition approach will allow us to implement the thermal coupling problem in a master-slave algorithm.

The model presented in Section 2 is based on the solution of a thermal problem in the whole domain $\Omega$ that includes the solid subdomain $\Omega_{\mathrm{S}}$ and the fluid subdomain $\Omega_{\mathrm{F}}$ separated by the interface $\Gamma_{\mathrm{SF}}$ which could be, in principle, a moving surface. The differential operators that describe

\footnotetext{
*Correspondence to: Javier Principe, CIMNE, Universitat Politècnica de Catalunya, Jordi Girona 1-3, Edifici C1, 08034 Barcelona, Spain.

†E-mail: principe@cimne.upc.edu
} 
the evolution of the temperature $(\vartheta)$ are different as a result of different physics and they depend on other variables that describe the state of each medium. On each subdomain the thermal problem could be coupled to other differential problems depending on the physical model used for the fluid and solid. In the first case we may have a compressible flow or an incompressible one, a mix of species, chemical reactions, etc. In the second case we may have a purely thermal problem, a thermomechanical one or even a thermo-hygro-mechanical one as in [2]. Any model can be used on each subdomain but we will assume that the coupling between the fluid and solid is only due to heat exchange. In the case in which mechanical problems are solved on each subdomain, this assumption is equivalent to assume that

1. the velocities of the solid medium are small and

2. the mechanical traction produced by the fluid on the solid is small.

It will be shown in Section 2 how these conditions result in the uncoupling of the mechanical problems. We will also assume that the displacements of the solid medium are small enough to consider the interface $\Gamma_{\mathrm{SF}}$ fixed.

The numerical approximation of the fluid and solid problems is in general different. One important feature of our approach is that different numerical approximations could be used to solve each problem. In the case of the fluid we use a stabilized finite element formulation based on the subgrid scale concept. Each field is decomposed into a resolvable and a subgrid scale

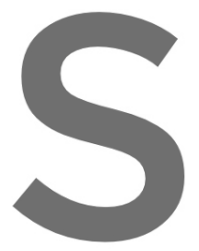
part according to the scale is taken into acco convection-dominate which would lead to Galerkin approximation in Section 3 but we emphasize
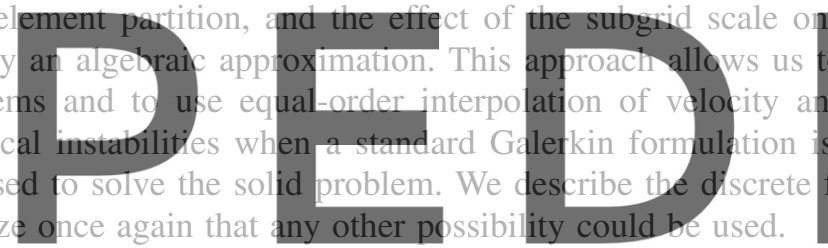

The possibility of using different models and different discrete approximations is not only

type. This leads to a non-overlapping domain decomposition problem that we implement in an iteration-by-subdomain strategy. The solution of each thermal probiem, in the fluid and in the solid regions, and the transmission of boundary conditions from one domain to the other is done by a relatively small master code. This code, developed following the MPI 2 standard, is in charge of managing the subdomain iterative coupling and the time-marching loops. In this way, each dedicated code acts as a slave and can be updated separately as only minor modifications are needed to change the information with the master code. These implementation aspects are described in Section 4. The domain decomposition framework for the thermal coupling described, together with its implementation aspects and an interpretation of the use of wall function approaches, is the main contribution of this work.

Finally, the approach is illustrated in a simple one-dimensional example and is applied to the simulation of a fire in a tunnel in Section 5. Some conclusions are drawn in Section 6.

\section{CONTINUOUS PROBLEM}

We consider a thermal problem in a domain $\Omega$ composed of two subdomains $\Omega_{\mathrm{S}}$ and $\Omega_{\mathrm{F}}$, as illustrated in Figure 1 (left and center). First, we present the problem in the whole domain considering 


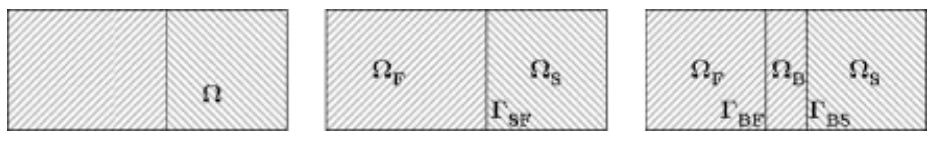

Figure 1. Domain of the problem.

discontinuous physical properties, which include the density $(\rho)$, the specific heat $\left(c_{p}\right)$ and the diffusion coefficient ( $\kappa=k / \rho c_{p}$, where $k$ is the thermal conductivity), as well as a velocity field (v). This velocity field will be assumed to be solution of a mechanical problem defined also in the whole domain and having also discontinuous properties. The constitutive relations in the fluid and in the solid are different, the former relating the stress tensor $(\boldsymbol{\sigma})$ to the velocity gradients and the latter relating the stress tensor to the deformation gradient.

Once the problem in the whole domain has been expressed, we will present two different strategies for a domain decomposition approach to this problem. The first strategy presented consists of a standard non-overlapping domain decomposition of the problem into the fluid and solid subdomains. We will assume that the mechanical problem in the solid does not depend on that in the fluid, in a sense to be made precise later on. We will refer to this approach as the full resolution strategy.

The second strategy consists of a non-overlapping domain decomposition of the problem in

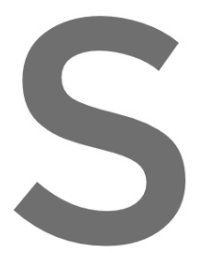
three subdomains, one (right). One of the fluid the other will be the the problem of the approach. An approxim wall function and an this second approach will also involve two subdomains. We again assume that the mechanical

\subsection{Problem definition in the whole domain}

2.1.1. Strong form of the problem. The problem to be solved in $\Omega$, an open domain in $\mathbb{R}^{d}(d=2,3$ is the number of space dimensions) during the time interval $\left(0, t_{\mathrm{f}}\right)$ is described by the equations of continuous media. Although a complex model can be used [2] the set of equations always contain an energy conservation statement that, under suitable assumptions, reads

$$
\rho c_{p}\left(\partial_{t} \vartheta+\mathbf{v} \cdot \nabla \vartheta\right)+\nabla \cdot \mathbf{q}=Q \quad \text { in } \Omega \times\left(0, t_{\mathrm{f}}\right)
$$

where $Q$ is the external source of energy source per unit of mass and $\mathbf{q}$ is the internal heat flux vector. The velocity field $\mathbf{v}$ is the solution of a mechanical problem of the form

$$
\rho\left(\partial_{t} \mathbf{v}+\mathbf{v} \cdot \nabla \mathbf{v}\right)-\nabla \cdot \boldsymbol{\sigma}=\rho \mathbf{g} \quad \text { in } \Omega \times\left(0, t_{\mathrm{f}}\right)
$$

where $\mathbf{g}$ is the external source of momentum per unit of mass and $\boldsymbol{\sigma}$ the internal stress tensor. The parameters present in these equations $\left(\rho\right.$ and $c_{p}$ ) may be discontinuous across the surface $\Gamma_{\mathrm{SF}}$. The constitutive equation for the internal heat flux is

$$
\mathbf{q}=-k \nabla \vartheta
$$


where $k$ may also be discontinuous across $\Gamma_{\mathrm{SF}}$. The constitutive equation for the internal stress tensor will in general be different in both regions. In the case of the solid it will be related to the deformation tensor. In the case of an incompressible fluid it will be related to the velocity gradient and to another variable, the pressure $(p)$, that will involve the solution of another equation, the conservation of mass. The problem must be supplemented with appropriate boundary and initial conditions, which can be expressed as

$$
\begin{gathered}
\vartheta=\vartheta_{0} \quad \text { in } \Omega \times\{0\} \\
\vartheta=\vartheta_{\mathrm{D}} \quad \text { on } \Gamma_{\mathrm{D}}^{\vartheta} \times\left(0, t_{\mathrm{f}}\right) \\
\mathbf{n} \cdot \mathbf{q}=q_{\mathrm{N}} \quad \text { on } \Gamma_{\mathrm{N}}^{\vartheta} \times\left(0, t_{\mathrm{f}}\right)
\end{gathered}
$$

where $\Gamma_{\mathrm{D}}^{\vartheta}\left(\Gamma_{\mathrm{N}}^{\vartheta}\right)$ represent the part of the domain boundary where Dirichlet (Neumann) boundary conditions for the temperature are prescribed, $\partial \Omega=\Gamma_{\mathrm{D}}^{\vartheta} \cup \Gamma_{\mathrm{N}}^{\vartheta}$ and $\mathbf{n}$ is the exterior normal to the domain $\Omega$. In turn, the initial and boundary conditions for the mechanical problem depend on the constitutive relation considered. In the case of a solid, they are usually expressed in terms of the displacement and in the case of the fluid in terms of the velocity. The purpose of expressing the mechanical problem is to clearly specify the conditions under which it is uncoupled. Nevertheless,

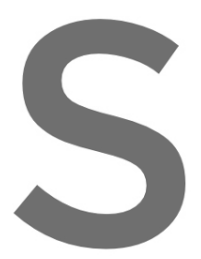
we will consider the weak form and the numer
2.1.2. Weak form of the problem. As usual we
order derivatives belong to $L^{2}(\Omega)$, the spare of s
and by $(u, v) \Omega$ the scalar product in $L^{2}(\Omega)$. Th boundary conditions will be denoted by $V^{\vartheta}\left(V^{0}\right.$

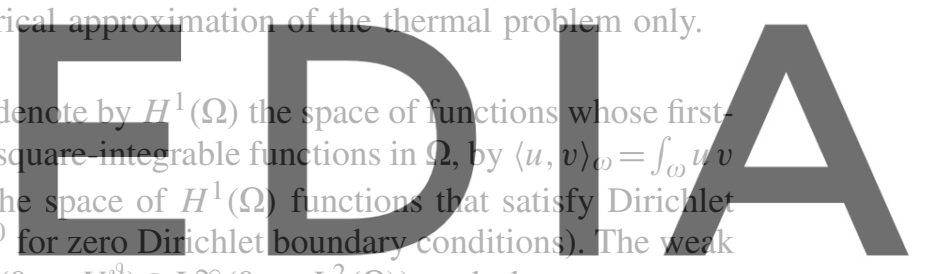
form of the problem consists in finding $\vartheta \in L^{2}\left(0, t_{\mathrm{f}} ; V^{\vartheta}\right) \cap L^{\infty}\left(0, t_{\mathrm{f}} ; L^{2}(\Omega)\right)$ such that

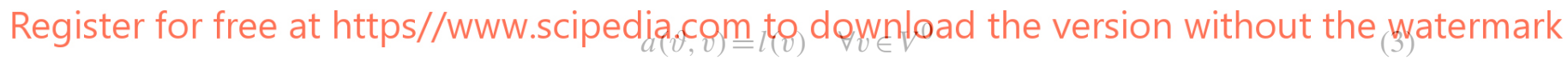

where $L^{2}\left(0, t_{\mathrm{f}} ; V^{\vartheta}\right)$ is the set of functions whose norm in $V^{\vartheta}$ (which is the norm in $H^{1}(\Omega)$ ), is square integrable in time, and $L^{\infty}\left(0, t_{\mathrm{f}} ; L^{2}(\Omega)\right)$ the set of functions whose norm in $L^{2}(\Omega)$ is bounded in time. The bilinear form $a$ and the linear form $l$ are defined as

$$
a(\vartheta, v):=\left(\rho c_{p} \partial_{t} \vartheta, v\right)_{\Omega}+\left(\rho c_{p} \mathbf{v} \cdot \nabla \vartheta, v\right)_{\Omega}+(k \nabla \vartheta, \nabla v)_{\Omega}
$$

and

$$
l(v):=\langle Q, v\rangle_{\Omega}+\left\langle q_{\mathrm{N}}, v\right\rangle_{\Gamma_{\mathrm{N}}^{\vartheta}}
$$

respectively (in fact, $a$ is affine in the first argument, but we will omit this precision in the following).

\subsection{The full resolution strategy}

A standard non-overlapping domain decomposition of the problem is obtained by splitting the domain $\Omega$ into the solid and fluid subdomains, $\Omega_{\mathrm{S}}$ and $\Omega_{\mathrm{F}}$, as illustrated in Figure 1 (center), where the notation that we will use is indicated. 
2.2.1. Strong form of the problem. The strong form of the problem consists in finding temperatures $\vartheta_{\mathrm{S}}$ and $\vartheta_{\mathrm{F}}$, as well as velocities $\mathbf{v}_{\mathrm{F}}$ (in the fluid) and displacements $\mathbf{u}_{\mathrm{S}}$ (in the solid), such that

$$
\begin{gathered}
\rho_{\mathrm{S}} c_{p \mathrm{~S}} \partial_{t} \vartheta_{\mathrm{S}}+\nabla \cdot \mathbf{q}_{\mathrm{S}}=Q_{\mathrm{S}} \quad \text { in } \Omega_{\mathrm{S}} \times\left(0, t_{\mathrm{f}}\right) \\
-\nabla \cdot \boldsymbol{\sigma}_{\mathrm{S}}=\rho \mathbf{g} \quad \text { in } \Omega_{\mathrm{S}} \times\left(0, t_{\mathrm{f}}\right)
\end{gathered}
$$

and

$$
\begin{gathered}
\rho_{\mathrm{F}} c_{p \mathrm{~F}}\left(\partial_{t} \vartheta_{\mathrm{F}}+\mathbf{v}_{\mathrm{F}} \cdot \nabla \vartheta_{\mathrm{F}}\right)+\nabla \cdot \mathbf{q}_{\mathrm{F}}=Q_{\mathrm{F}} \quad \text { in } \Omega_{\mathrm{F}} \times\left(0, t_{\mathrm{f}}\right) \\
\rho_{\mathrm{F}}\left(\partial_{t} \mathbf{v}_{\mathrm{F}}+\mathbf{v}_{\mathrm{F}} \cdot \nabla \mathbf{v}_{\mathrm{F}}\right)-\nabla \cdot \boldsymbol{\sigma}_{\mathrm{F}}=\rho \mathbf{g} \quad \text { in } \Omega_{\mathrm{F}} \times\left(0, t_{\mathrm{f}}\right)
\end{gathered}
$$

Here we have used Assumption 1 of Section 1 to neglect the terms containing the velocity of the solid. For the sake of simplicity in the exposition, accelerations have also been neglected (quasistatic problem). The conditions to be satisfied at the interface are the continuity of the temperatures and velocities as well as the normal components of heat fluxes and tractions that read
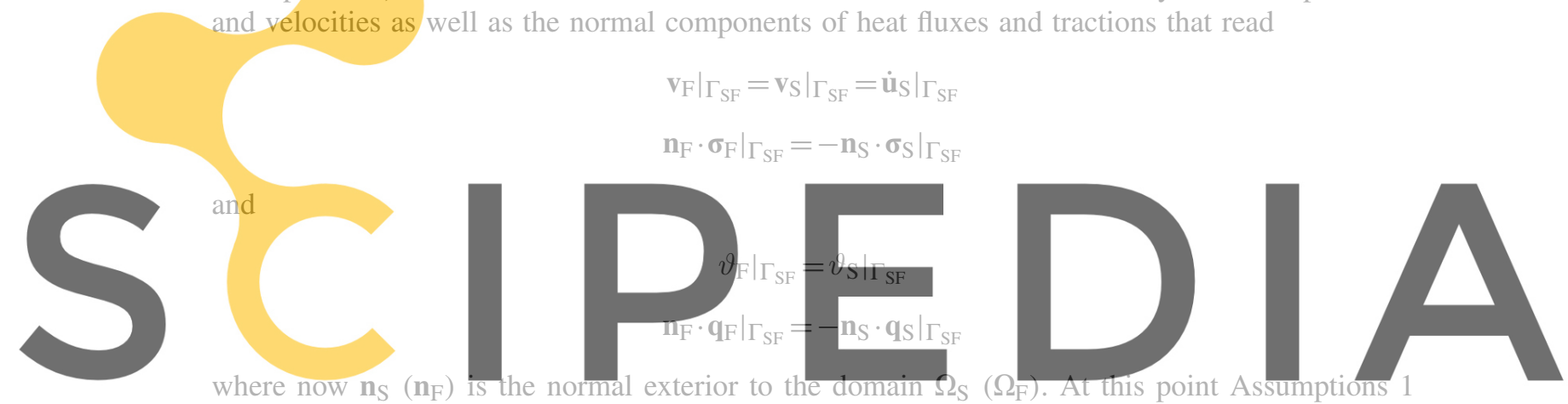

and 2 of Section 1 yield $\dot{u}_{S} \approx 0$ and $\left.\mathrm{n}_{\mathrm{F}} \cdot \sigma_{\mathrm{F}}\right|_{\Gamma_{\mathrm{SF}}} \approx 0$, respectively. With these approximations the Register forifreenatchitdpishswowbuscipedia.com to download the version without the watermark

$$
\begin{gathered}
\left.\mathbf{v}_{\mathrm{F}}\right|_{\Gamma_{\mathrm{SF}}}=0 \\
\left.\mathbf{n}_{\mathrm{S}} \cdot \boldsymbol{\sigma}_{\mathrm{S}}\right|_{\Gamma_{\mathrm{SF}}}=\mathbf{0}
\end{gathered}
$$

and

$$
\begin{gathered}
\left.\vartheta_{\mathrm{F}}\right|_{\Gamma_{\mathrm{SF}}}=\left.\vartheta_{\mathrm{S}}\right|_{\Gamma_{\mathrm{SF}}} \\
\left.\mathbf{n}_{\mathrm{F}} \cdot \mathbf{q}_{\mathrm{F}}\right|_{\Gamma_{\mathrm{SF}}}=-\left.\mathbf{n}_{\mathrm{S}} \cdot \mathbf{q}_{\mathrm{S}}\right|_{\Gamma_{\mathrm{SF}}}
\end{gathered}
$$

and therefore the mechanical problems are uncoupled.

2.2.2. Weak form of the problem. Let us introduce the bilinear forms $a_{\mathrm{S}}$ defined on the solid subdomain $\Omega_{\mathrm{S}}$ as

$$
a_{\mathrm{S}}(\vartheta, v):=\left(\rho_{\mathrm{S}} c_{p \mathrm{~S}} \partial_{t} \vartheta_{\mathrm{S}}, v\right)_{\Omega_{\mathrm{S}}}+\left(k_{\mathrm{S}} \nabla \vartheta_{\mathrm{S}}, \nabla v\right)_{\Omega_{\mathrm{S}}}
$$

and on the fluid subdomain $\Omega_{\mathrm{F}}$ as

$$
a_{\mathrm{F}}(\vartheta, v):=\left(\rho_{\mathrm{F}} c_{p \mathrm{~F}} \partial_{t} \vartheta_{\mathrm{F}}, v\right)_{\Omega_{\mathrm{F}}}+\left(\rho_{\mathrm{F}} c_{p \mathrm{~F}} \mathbf{v}_{\mathrm{F}} \cdot \nabla \vartheta_{\mathrm{F}}, v\right)_{\Omega_{\mathrm{F}}}+\left(k_{\mathrm{F}} \nabla \vartheta_{\mathrm{F}}, \nabla v\right)_{\Omega_{\mathrm{F}}}
$$


We also introduce the linear forms

$$
l_{\mathrm{S}}(v):=\left\langle Q_{\mathrm{S}}, v\right\rangle_{\Omega_{\mathrm{S}}}+\left\langle q_{\mathrm{N}}, v\right\rangle_{\Gamma_{\mathrm{NS}}^{\vartheta}}
$$

and

$$
l_{\mathrm{F}}(v):=\left\langle Q_{\mathrm{F}}, v\right\rangle_{\Omega_{\mathrm{F}}}+\left\langle q_{\mathrm{N}}, v\right\rangle_{\Gamma_{\mathrm{NF}}^{\vartheta}}
$$

The weak form of the problem consists in finding

$$
\begin{aligned}
& \vartheta_{\mathrm{S}} \in L^{2}\left(0, t_{\mathrm{f}} ; V_{\mathrm{S}}^{\vartheta}\right) \cap L^{\infty}\left(0, t_{\mathrm{f}} ; L^{2}\left(\Omega_{\mathrm{S}}\right)\right) \\
& \vartheta_{\mathrm{F}} \in L^{2}\left(0, t_{\mathrm{f}} ; V_{\mathrm{F}}^{\vartheta}\right) \cap L^{\infty}\left(0, t_{\mathrm{f}} ; L^{2}\left(\Omega_{\mathrm{F}}\right)\right)
\end{aligned}
$$

such that

$$
\begin{array}{r}
a_{\mathrm{S}}(\vartheta, v)-\left\langle k_{\mathrm{S}} \mathbf{n}_{\mathrm{S}} \cdot \nabla \vartheta_{\mathrm{S}}, v\right\rangle_{\Gamma_{\mathrm{SF}}}=l_{\mathrm{S}}(v) \quad \forall v \in V_{\mathrm{S}}^{0} \\
a_{\mathrm{F}}(\vartheta, v)-\left\langle k_{\mathrm{F}} \mathbf{n}_{\mathrm{F}} \cdot \nabla \vartheta_{\mathrm{F}}, v\right\rangle_{\Gamma_{\mathrm{SF}}}=l_{\mathrm{F}}(v) \quad \forall v \in V_{\mathrm{F}}^{0} \\
\vartheta_{\mathrm{F}}=\vartheta_{\mathrm{S}} \quad \text { on } \Gamma_{\mathrm{SF}}
\end{array}
$$
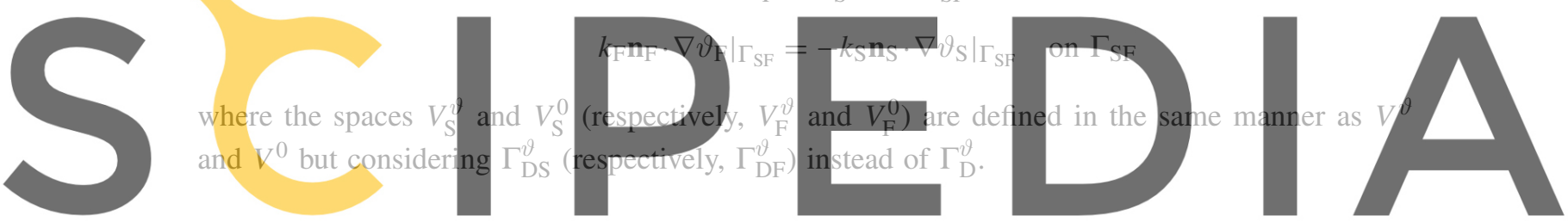

2.3. The wall function strategy

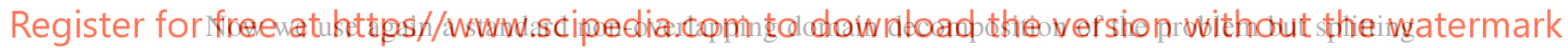

the domain $\Omega$ into the solid subdomain $\Omega_{\mathrm{S}}$, a boundary layer in the fluid $\Omega_{\mathrm{B}}$ and the rest of the

fluid subdomain $\Omega_{\mathrm{F}}$, as illustrated in Figure 1 (right). In addition, let $\Gamma_{\mathrm{SB}}$ be the interface between

$\Omega_{\mathrm{S}}$ and $\Omega_{\mathrm{B}}$, and $\Gamma_{\mathrm{BF}}$ be the interface between $\Omega_{\mathrm{B}}$ and $\Omega_{\mathrm{F}}$. Apart from the equations given in the previous subsection for the solid and the fluid subdomains, we now need to solve the problem on the boundary layer subdomain, which consists in finding a temperature $\vartheta_{\mathrm{B}}$ and a velocity $\mathbf{v}_{\mathrm{B}}$ such that

$$
\begin{gathered}
\rho c_{p}\left(\partial_{t} \vartheta_{\mathrm{B}}+\mathbf{v}_{\mathrm{B}} \cdot \nabla \vartheta_{\mathrm{B}}\right)+\nabla \cdot \mathbf{q}_{\mathrm{B}}=Q_{\mathrm{B}} \\
\rho\left(\partial_{t} \mathbf{v}_{\mathrm{B}}+\mathbf{v}_{\mathrm{B}} \cdot \nabla \mathbf{v}_{\mathrm{B}}\right)-\nabla \cdot \boldsymbol{\sigma}_{\mathrm{B}}=\rho \mathbf{g}
\end{gathered}
$$

Under the same assumptions as in the previous subsection the interface conditions are

$$
\begin{gathered}
\left.\mathbf{v}_{\mathrm{B}}\right|_{\Gamma_{\mathrm{SB}}}=\mathbf{0} \\
\left.\mathbf{n}_{\mathrm{S}} \cdot \boldsymbol{\sigma}_{\mathrm{S}}\right|_{\Gamma_{\mathrm{SB}}}=\mathbf{0} \\
\left.\vartheta_{\mathrm{B}}\right|_{\Gamma_{\mathrm{SB}}}=\left.\vartheta_{\mathrm{S}}\right|_{\Gamma_{\mathrm{SB}}} \\
\left.\mathbf{n}_{\mathrm{B}} \cdot \mathbf{q}_{\mathrm{B}}\right|_{\Gamma_{\mathrm{SB}}}=-\left.\mathbf{n}_{\mathrm{S}} \cdot \mathbf{q}_{\mathrm{S}}\right|_{\Gamma_{\mathrm{SB}}}
\end{gathered}
$$


and

$$
\begin{gathered}
\left.\mathbf{v}_{\mathrm{F}}\right|_{\Gamma_{\mathrm{BF}}}=\left.\mathbf{v}_{\mathrm{B}}\right|_{\Gamma_{\mathrm{BF}}} \\
\left.\mathbf{n}_{\mathrm{F}} \cdot \boldsymbol{\sigma}_{\mathrm{F}}\right|_{\Gamma_{\mathrm{BF}}}=-\left.\mathbf{n}_{\mathrm{B}} \cdot \boldsymbol{\sigma}_{\mathrm{B}}\right|_{\Gamma_{\mathrm{BF}}} \\
\left.\vartheta_{\mathrm{F}}\right|_{\Gamma_{\mathrm{BF}}}=\left.\vartheta_{\mathrm{B}}\right|_{\Gamma_{\mathrm{BF}}} \\
\left.\mathbf{n}_{\mathrm{F}} \cdot \mathbf{q}_{\mathrm{F}}\right|_{\Gamma_{\mathrm{BF}}}=-\left.\mathbf{n}_{\mathrm{B}} \cdot \mathbf{q}_{\mathrm{B}}\right|_{\Gamma_{\mathrm{BF}}}
\end{gathered}
$$

If the boundary layer is of constant width, one may assume that its normal satisfies

$$
\left.\mathbf{n}_{\mathrm{B}}\right|_{\Gamma_{\mathrm{SB}}}=-\left.\mathbf{n}_{\mathrm{B}}\right|_{\Gamma_{\mathrm{BF}}}
$$

The problem on the boundary layer subdomain is now approximately solved using the wall function approach, which is described next.

2.3.1. Wall function revisited. The so-called wall function approach is a method for the approximate solution of the fluid mechanics problems with strong boundary layers. These boundary layers are removed from the computational domain and universal velocity profiles are used to define the boundary condition in terms of the boundary conditions on the solid surface, as shown in Figure 2.

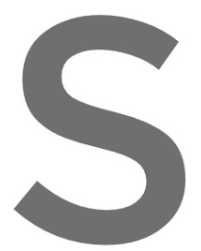

This approximated sol

(5) and (6) it is seen that this yir
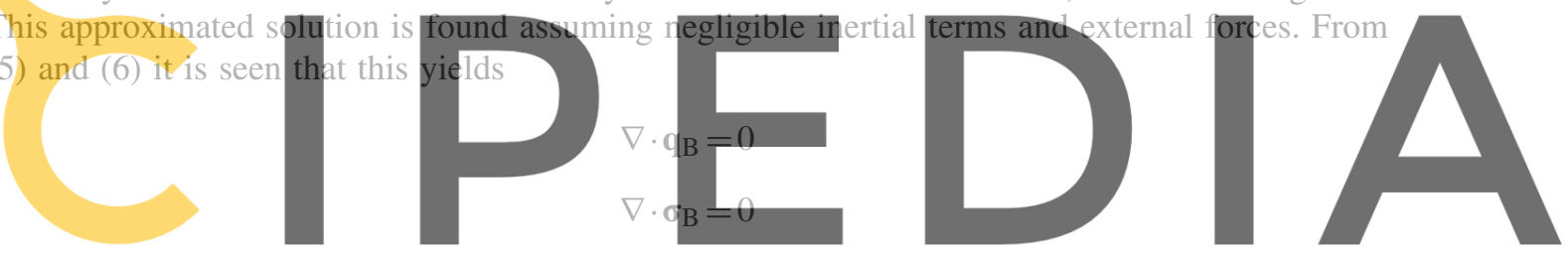

which imply constant stresses and heat fluxes across the boundary layer. Now, we denote as $q$ the

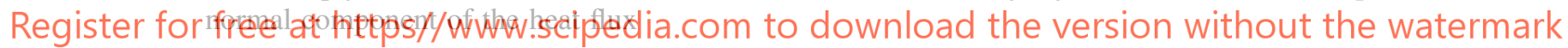

$$
q=\left.\mathbf{n}_{\mathrm{B}} \cdot \mathbf{q}_{\mathrm{B}}\right|_{\Gamma_{\mathrm{SB}}}
$$

and as $\mathbf{t}$ the tangential stress

$$
\mathbf{t}=\mathbf{n} \cdot \boldsymbol{\sigma}_{\mathrm{B}}-\left.\left(\mathbf{n} \cdot \boldsymbol{\sigma}_{\mathrm{B}} \cdot \mathbf{n}\right) \mathbf{n}\right|_{\Gamma_{\mathrm{SB}}}
$$

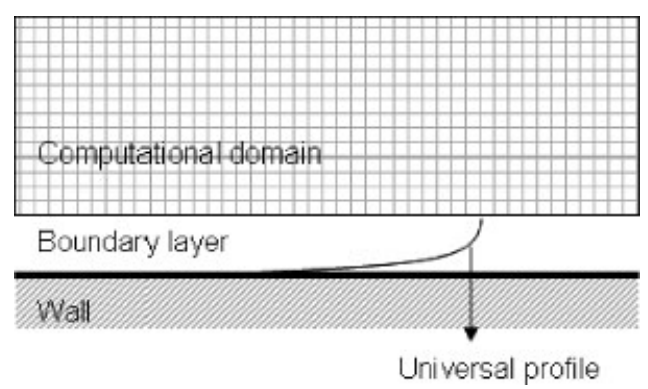

Figure 2. A wall function approach. 
evaluated at the wall. The mechanical problem is solved selecting a local coordinate system such that the first local direction is $\mathbf{t}$. If we denote by $\sigma$ the norm of the tangential component of the stress (not to be confused with the full stress tensor), by $u_{\mathrm{B}}$ the component of the velocity in this system and by $y$ the coordinate normal to the solid surface, the constitutive equations are

$$
\begin{aligned}
& q=\left(k+k^{\mathrm{t}}\right) \frac{\mathrm{d} \vartheta_{\mathrm{B}}}{\mathrm{d} y} \\
& \sigma=\left(\mu+\mu^{\mathrm{t}}\right) \frac{\mathrm{d} u_{\mathrm{B}}}{\mathrm{d} y}
\end{aligned}
$$

where $\mu^{\mathrm{t}}$ and $k^{\mathrm{t}}$ are the turbulent viscosity and conductivity. Given the turbulent viscosity and conductivity we can integrate (7) and (8) to obtain the velocity and temperature profiles. The definition of the turbulent viscosity and conductivity is the definition of the model we are using to approximate the problem and is based on experimental correlations [3]. One of these models is the one that results in the logarithmic profiles for the velocity and temperature. This model is based on the existence of a zone near the wall, called laminar sublayer, in which the velocity is small and as well the local Reynolds number. In the laminar sublayer the turbulent viscosity is neglected and we have
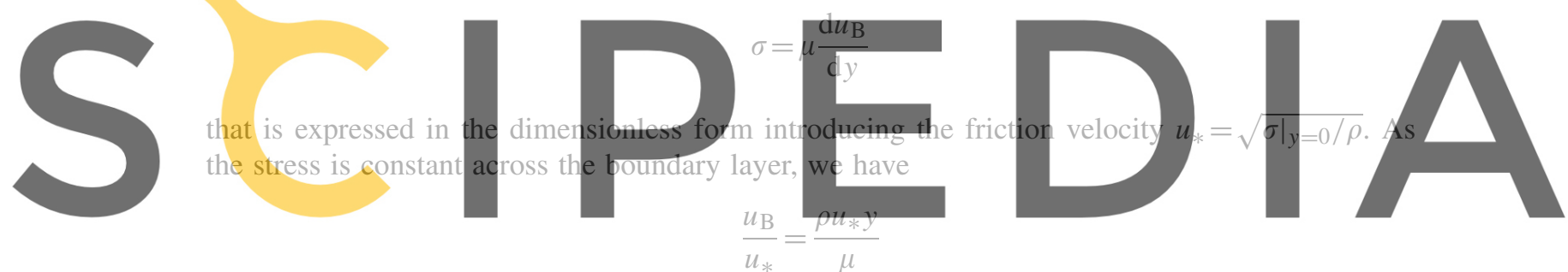

Register for free at https//www.scipedia.com to download the version without the watermark On the other hand, in the turbulent region we may approximate $\mu^{\mathrm{t}}=\rho \kappa y u_{*}, \kappa$ being the Von

Karman constant, and integrating (8) we have

$$
\frac{u_{\mathrm{B}}}{u_{*}}=\frac{u_{0}}{u_{*}}+\frac{1}{\kappa} \ln \left(\frac{y}{y_{0}}\right)
$$

where $y_{0}$ is the width of the laminar sublayer and $u_{0}$ the value of the velocity at this point. Defining the dimensionless velocity $u_{\mathrm{B}}^{+}=u_{\mathrm{B}} / u_{*}$ and distance $y^{+}=\rho y u^{*} / \mu$ and taking $y_{0}^{+}=11.6$ the final solution reads

$$
u_{\mathrm{B}}^{+}= \begin{cases}y^{+} & \text {if } y^{+}<y_{0}^{+} \\ \frac{1}{\kappa} \ln \left(y^{+}\right)+5.5 & \text { if } y^{+} \geqslant y_{0}^{+}\end{cases}
$$

In the same manner, integrating (7) we arrive at

$$
\vartheta_{\mathrm{B}}^{+}= \begin{cases}\operatorname{Pry}^{+} & \text {if } y^{+}<y_{0}^{+} \\ \operatorname{Pr}^{\mathrm{t}}\left[\frac{1}{\kappa} \ln \left(y^{+}\right)+P_{\theta}\right] & \text { if } y^{+} \geqslant y_{0}^{+}\end{cases}
$$


where $\operatorname{Pr}:=\rho \kappa / \mu$ is the Prandtl number, $\operatorname{Pr}^{\mathrm{t}}$ is the turbulent Prandtl number (which is part of the constitutive model), $P_{\theta}$ a function that gives the temperature jump across the laminar sublayer and the dimensionless temperature is defined as

$$
\vartheta_{\mathrm{B}}^{+}=-\frac{\rho c_{p} u_{*}}{q}\left(\vartheta-\left.\vartheta_{\mathrm{B}}\right|_{\Gamma_{\mathrm{SB}}}\right)
$$

2.3.2. Strong form of the problem. Having an analytical solution to the problem in the boundary layer domain we can rewrite the complete problem in terms of two subdomains, the fluid (excluding the boundary layer) and the solid. To this end, let us remark that the solution of the thermal problem obtained using the wall function method is a constant heat flux and therefore

$$
q=\left.n_{\mathrm{S}} \cdot q_{\mathrm{S}}\right|_{\Gamma_{\mathrm{SB}}}=-\left.\mathrm{n}_{\mathrm{B}} \cdot q_{\mathrm{B}}\right|_{\Gamma_{\mathrm{SB}}}=\left.\mathrm{n}_{\mathrm{B}} \cdot \mathrm{q}_{\mathrm{B}}\right|_{\Gamma_{\mathrm{BF}}}=-\left.\mathrm{n}_{\mathrm{F}} \cdot q_{\mathrm{F}}\right|_{\Gamma_{\mathrm{BF}}}
$$

This flux is proportional to the temperature jump across the layer

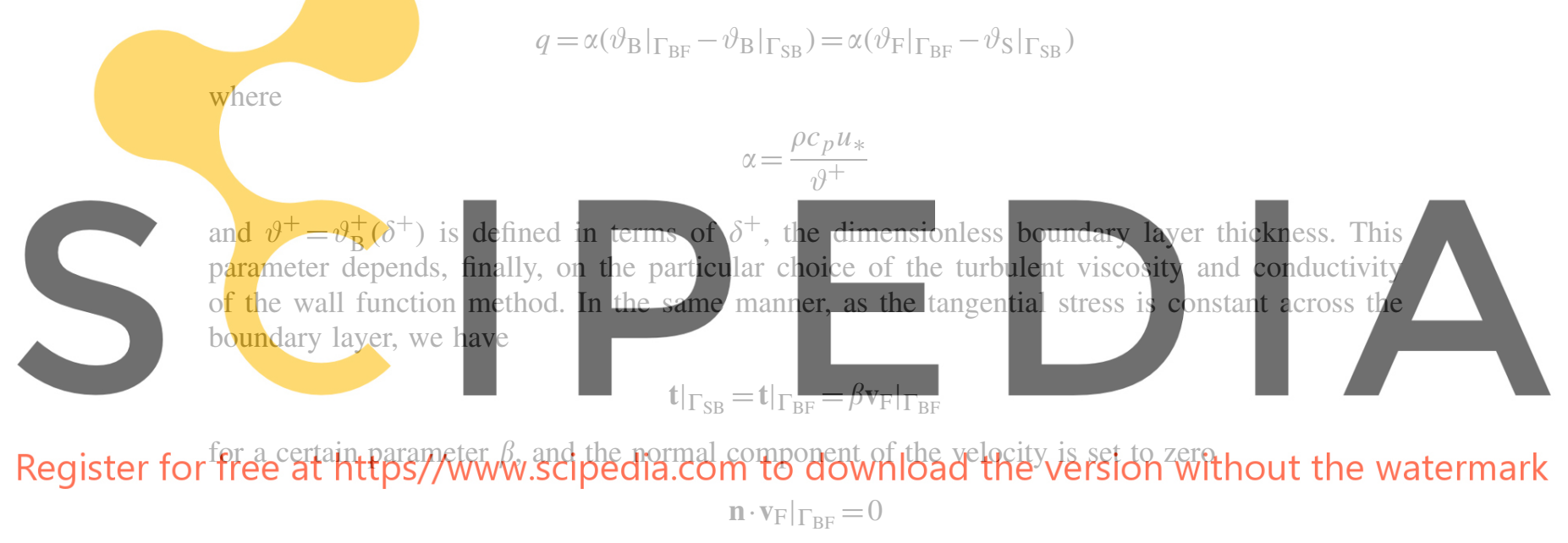

We can finally state the strong form of the problem as finding $\vartheta_{\mathrm{S}}$ and $\vartheta_{\mathrm{F}}$, as well as $\mathbf{u}_{\mathrm{S}}$ and $\mathbf{v}_{\mathrm{F}}$, such that

$$
\begin{gathered}
\rho_{\mathrm{S}} c_{p \mathrm{~S}} \partial_{t} \vartheta_{\mathrm{S}}+\nabla \cdot \mathbf{q}_{\mathrm{S}}=Q_{\mathrm{S}} \quad \text { in } \Omega_{\mathrm{S}} \times\left(0, t_{\mathrm{f}}\right) \\
-\nabla \cdot \boldsymbol{\sigma}_{\mathrm{S}}=\rho \mathbf{g} \quad \text { in } \Omega_{\mathrm{S}} \times\left(0, t_{\mathrm{f}}\right)
\end{gathered}
$$

and

$$
\begin{gathered}
\rho_{\mathrm{S}} c_{p \mathrm{~S}}\left(\partial_{t} \vartheta_{\mathrm{F}}+\mathbf{v}_{\mathrm{F}} \cdot \nabla \vartheta_{\mathrm{F}}\right)+\nabla \cdot \mathbf{q}_{\mathrm{F}}=Q_{\mathrm{F}} \quad \text { in } \Omega_{\mathrm{F}} \times\left(0, t_{\mathrm{f}}\right) \\
\rho_{\mathrm{S}}\left(\partial_{t} \mathbf{v}_{\mathrm{F}}+\mathbf{v}_{\mathrm{F}} \cdot \nabla \mathbf{v}_{\mathrm{F}}\right)-\nabla \cdot \boldsymbol{\sigma}_{\mathrm{F}}=\rho \mathbf{g} \quad \text { in } \Omega_{\mathrm{F}} \times\left(0, t_{\mathrm{f}}\right)
\end{gathered}
$$

and now the interface conditions become

$$
\begin{gathered}
\left.\mathbf{t}\right|_{\Gamma_{\mathrm{BF}}}=\left.\beta \mathbf{v}_{\mathrm{F}}\right|_{\Gamma_{\mathrm{BF}}} \\
\left.\mathbf{n}_{\mathrm{F}} \cdot \mathbf{v}_{\mathrm{F}}\right|_{\Gamma_{\mathrm{BF}}}=0 \\
\left.\mathbf{n}_{\mathrm{S}} \cdot \boldsymbol{\sigma}_{\mathrm{S}}\right|_{\Gamma_{\mathrm{SB}}}=\mathbf{0}
\end{gathered}
$$


and

$$
\begin{aligned}
q & =\left.\mathbf{n}_{\mathrm{S}} \cdot \mathbf{q}_{\mathrm{S}}\right|_{\Gamma_{\mathrm{SB}}}=-\left.\mathbf{n}_{\mathrm{B}} \cdot \mathbf{q}_{\mathrm{B}}\right|_{\Gamma_{\mathrm{SB}}}=\left.\mathbf{n}_{\mathrm{B}} \cdot \mathbf{q}_{\mathrm{B}}\right|_{\Gamma_{\mathrm{BF}}}=-\left.\mathbf{n}_{\mathrm{F}} \cdot \mathbf{q}_{\mathrm{F}}\right|_{\Gamma_{\mathrm{BF}}} \\
& =\alpha\left(\left.\vartheta_{\mathrm{B}}\right|_{\Gamma_{\mathrm{BF}}}-\left.\vartheta_{\mathrm{B}}\right|_{\Gamma_{\mathrm{SB}}}\right)=\alpha\left(\left.\vartheta_{\mathrm{F}}\right|_{\Gamma_{\mathrm{BF}}}-\left.\vartheta_{\mathrm{S}}\right|_{\Gamma_{\mathrm{SB}}}\right)
\end{aligned}
$$

Finally, assuming the boundary layer to be thin, we can express the final approximation as

$$
\begin{aligned}
q & =\left.\mathbf{n}_{\mathrm{S}} \cdot \mathbf{q}_{\mathrm{S}}\right|_{\Gamma_{\mathrm{SF}}}=-\left.\mathbf{n}_{\mathrm{F}} \cdot \mathbf{q}_{\mathrm{F}}\right|_{\Gamma_{\mathrm{SF}}} \\
& =\alpha\left(\left.\vartheta_{\mathrm{F}}\right|_{\Gamma_{\mathrm{SF}}}-\left.\vartheta_{\mathrm{S}}\right|_{\Gamma_{\mathrm{SF}}}\right)
\end{aligned}
$$

which is a surface-convection-type boundary condition. As in [4], we have derived an expression for $\alpha$ based on the physical model being used (with a completely different meaning with respect to the mentioned reference).

\subsubsection{Weak form of the problem. Using the notation of the previous subsection, the weak form}

\section{of the problem consists in finding}
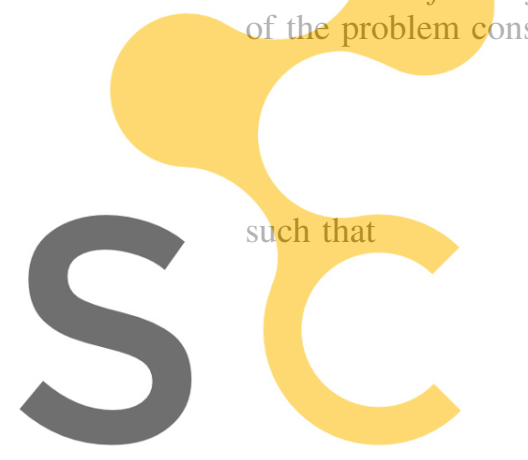

$$
\begin{aligned}
& \vartheta_{\mathrm{S}} \in L^{2}\left(0, t_{\mathrm{f}} ; V_{\mathrm{S}}^{\vartheta}\right) \cap L^{\infty}\left(0, t_{\mathrm{f}} ; L^{2}\left(\Omega_{\mathrm{S}}\right)\right) \\
& \vartheta_{\mathrm{F}} \in L^{2}\left(0, t_{\mathrm{f}} ; V_{\mathrm{F}}^{\vartheta}\right) \cap L^{\infty}\left(0, t_{\mathrm{f}} ; L^{2}\left(\Omega_{\mathrm{F}}\right)\right)
\end{aligned}
$$
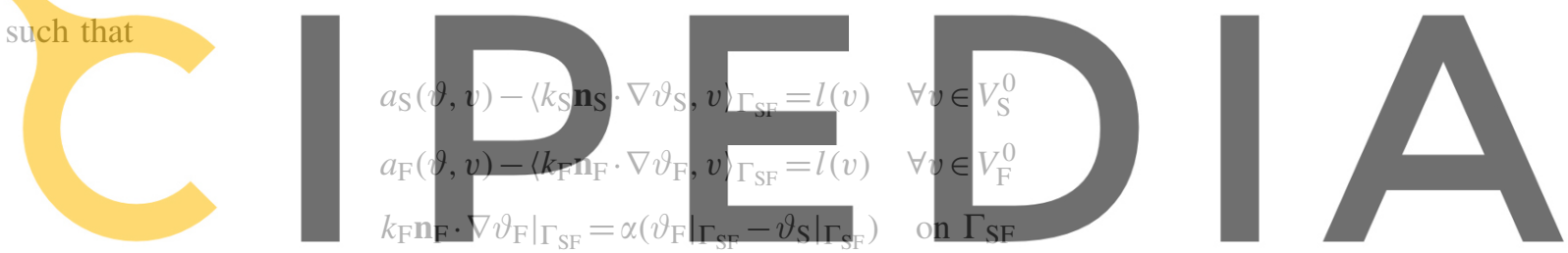

\section{Register for free at https//www.scipedia.com to download the version without the watermark Comparing the weak form of this problem to (4) the only difference is a jump on the temperature proportional to the heat flux between domains. Our derivation allows us to give an interpretation} to the surface convection coefficient $\alpha$ in terms of the wall function model used on the boundary layer subdomain.

\section{NUMERICAL APPROXIMATION}

Three different continuous problems have been described in Section 2 but the first one, that consists of the solution of a global problem in the whole domain, was presented to define the problem we are facing and has not been actually implemented. The other two possibilities imply the solution of local thermal problems as well as local mechanical problems for the fluid and the solid. In this section we present the numerical approximation to the problem and we will concentrate on the thermal problem only. In the first subsection we will present the finite element discretization of the problem considering generically the domain $\Omega$. This approximation could be applied on the whole domain but will be actually applied on each subdomain. A similar scheme is used to solve the mechanical problem on the fluid. Details on the finite element approximation to the Navier-Stokes equation can be found in [5-7]. In the second subsection we describe an iterative strategy to solve the global thermal problem iteratively solving local problems on each subdomain. 


\subsection{Finite element approximation}

The Galerkin finite element approximation of this problem is standard. Based on a partition of the domain $\mathcal{P}_{h}=\{K\}$ in $n_{\mathrm{el}}$ elements $K$, the space $V^{\vartheta}$ where the temperature is sought is approximated by a finite-dimensional space $V_{h}^{\vartheta}$ (built using polynomials). If the space of test functions $V^{0}$ is approximated by $V_{h}^{0}$, defined in a similar manner, the semi-discrete problem consists in finding $\vartheta_{h} \in L^{2}\left(0, t_{\mathrm{f}} ; V_{h}^{\vartheta}\right)$ such that

$$
a\left(\vartheta_{h}, v_{h}\right)=l\left(v_{h}\right) \quad \forall v_{h} \in V_{h}^{0}
$$

It is well known that this formulation is unstable when the convection dominates and therefore we employ a stabilized finite element formulation based on the subgrid scale method with an algebraic approximation to the subscales [8]. Details of the formulation are given in [6]. Only the final discrete problem will be presented here.

The time discretization of the problem will be performed using the generalized trapezoidal rule, that is to say, a finite difference scheme. Let us consider a uniform partition of the time interval $\left(0, t_{\mathrm{f}}\right)$ of size $\delta t$ and let us introduce the following notation:

$$
\begin{gathered}
f^{n+\theta}=\theta f^{n+1}+(1-\theta) f^{n} \\
\delta_{t} f^{n}=\left(f^{n+1}-f^{n}\right) / \delta t=\left(f^{n+\theta}-f^{n}\right) /(\theta \delta t)
\end{gathered}
$$

where $0<\theta \leqslant 1$. For $\theta=1$ we obtain the backward Euler scheme of first order, and for $\theta=\frac{1}{2}$ the Crank-Nicolson scheme of second order. Both are unconditionally stable. Let us define

$$
\begin{aligned}
a^{h}\left(\vartheta_{h}^{n+1}, v_{h}\right)= & \left(\rho^{n+\theta} c_{p}^{n+\theta} \delta_{t} \vartheta_{h}^{n}, v\right)_{\Omega}+\left(\rho^{n+\theta} c_{p}^{n+\theta} \mathbf{v}^{n+\theta} \cdot \nabla \vartheta_{h}^{n+\theta}, v\right)_{\Omega}+\left(k^{n+\theta} \nabla \vartheta_{h}^{n+\theta}, \nabla v_{h}\right)_{\Omega} \\
& +\left(\rho^{n+\theta} c_{p}^{n+\theta} \delta_{t} \widetilde{\vartheta}^{n}, v_{h}\right)_{\Omega}-\sum_{K}\left(\rho^{n+\theta} c_{p}^{n+\theta} \mathbf{v}^{n+\theta} \cdot \nabla v_{h}+k^{n+\theta} \nabla^{2} v_{h}, \widetilde{\vartheta}^{n+\theta}\right)_{K}
\end{aligned}
$$

where $\widetilde{\vartheta}^{n+1}$ is given by (see [6] for details)

- quasi-static subscales:

$$
\widetilde{\vartheta}^{n+\theta} \approx \tau^{n+\theta} R_{h}^{n+\theta}
$$

- dynamic subscales:

$$
\rho^{n+\theta} c_{p}^{n+\theta} \delta_{t} \widetilde{\vartheta}^{n}+\tau^{-1} \widetilde{\vartheta}^{n+\theta}=R_{h}^{n+\theta}
$$

The parameter $\tau$ appearing in these expressions is given by

$$
\tau=\left[c_{1} \frac{k}{h^{2}}+c_{2} \frac{\rho c_{p}\|\mathbf{v}\|}{h}\right]^{-1}
$$

The fully discrete problem consists of: For $n=1,2, \ldots$, find $\vartheta_{h}^{n+1} \in V_{h}^{\vartheta}$ such that

$$
a^{h}\left(\vartheta_{h}^{n+1}, v_{h}\right)=\left\langle q^{n+\theta}, v_{h}\right\rangle_{\Omega}+\left\langle q_{\mathrm{N}}^{n+\theta}, v_{h}\right\rangle_{\Gamma_{\mathrm{N}}^{\vartheta}} \quad \forall v_{h} \in V_{h}^{0}
$$




\subsection{Coupling strategy}

As mentioned before, we consider a geometric domain decomposition of the problem by means of a non-overlapping subdomain approach. Therefore, at each time step, we expect to construct the solution of the problem from the solution of local problems for the fluid and the structure using the interface conditions already described. This is carried out by iteratively solving local problems on each domain until convergence on the interface conditions is satisfied, that is to say, we use an iteration-by-subdomain strategy [9]. The choice of the boundary conditions of the local problems should be such that the interface conditions presented in Section 2 are satisfied when convergence is achieved. It is well known from the theory of domain decomposition methods that in the case of non-overlapping subdomains we can choose Dirichlet-Neumann (Robin), Neumann (Robin)-Dirichlet or Robin-Robin. Let us define $a_{\mathrm{S}}^{h}$ and $a_{\mathrm{F}}^{h}$ in the same manner as $a^{h}$ was defined in the previous subsection.

If we use the full resolution strategy and apply Dirichlet boundary conditions to the solid and Neumann boundary conditions to the fluid, which according to Giles and Roe et al. $[10,11]$ is the most stable option, the coupling algorithm can be expressed as: For each time step $n$ and each iteration $i$ find $\vartheta_{\mathrm{S}, h}^{n+1, i+1} \in V_{\mathrm{S}, h}^{\vartheta}$ and $\vartheta_{\mathrm{F}, h}^{n+1, i+1} \in V_{\mathrm{F}, h}^{\vartheta}$ such that

$$
\begin{gathered}
a_{\mathrm{S}}^{h}\left(\vartheta_{\mathrm{S}, h}^{n+1, i+1}, v_{h}\right)=\left\langle Q^{n+\theta}, v_{h}\right\rangle_{\Omega}+\left\langle q_{\mathrm{N}}^{n+\theta}, v_{h}\right\rangle_{\Gamma_{\mathrm{N}}^{\vartheta}} \\
a_{\mathrm{F}}^{h}\left(\vartheta_{\mathrm{F}, h}^{n+1, i+1}, v_{h}\right)=\left\langle Q^{n+\theta}, v_{h}\right\rangle_{\Omega}+\left\langle q_{\mathrm{N}}^{n+\theta}, v_{h}\right\rangle_{\Gamma_{\mathrm{N}}^{\vartheta}}-\left\langle k_{\mathrm{S}} \mathbf{n}_{\mathrm{S}} \cdot \nabla \vartheta_{\mathrm{S}, h}^{n+1, i}, v_{h}\right\rangle_{\Gamma_{\mathrm{SF}}}
\end{gathered}
$$

where $v_{h} \in V_{\mathrm{S}, h}^{0}$ in (9) and in $v_{h} \in V_{\mathrm{F}, h}^{0}$ in (10). Now it is understood that these spaces, $V_{\mathrm{S}, h}^{0}$ and $V_{\mathrm{S}, h}^{\vartheta}$, are constructed including $\Gamma_{\mathrm{SF}}$ in the Dirichlet part of the boundary in order to satisfy

$$
\vartheta_{\mathrm{S}, h}^{n+1, i+1}=\vartheta_{\mathrm{F}, h}^{n+1, k} \quad \text { on } \Gamma_{\mathrm{SF}}
$$

We can take $k=i+1$ or $k=i$. In the first case, the solution of this problem is sequential, that is, we solve first for the fluid and then for the solid, whereas in the second one it can be parallel.

If we use the wall function strategy, the coupling algorithm can be expressed as: For each time step and each iteration $i$ find $\vartheta_{\mathrm{S}, h}^{n+1, i+1} \in V_{\mathrm{S}, h}^{\vartheta}$ and $\vartheta_{\mathrm{F}, h}^{n+1, i+1} \in V_{\mathrm{F}, h}^{\vartheta}$ such that

$$
\begin{aligned}
& a_{\mathrm{S}}^{h}\left(\vartheta_{\mathrm{S}, h}^{n+1, i+1}, v_{h}\right)=\left\langle Q^{n+\theta}, v_{h}\right\rangle_{\Omega}+\left\langle q_{\mathrm{N}}^{n+\theta}, v_{h}\right\rangle_{\Gamma_{\mathrm{N}}^{\vartheta}}+\left\langle\alpha\left(\vartheta_{\mathrm{S}, h}^{n+1, i+1}-\vartheta_{\mathrm{F}, h}^{n+1, i}\right), v_{h}\right\rangle_{\Gamma_{\mathrm{SF}}} \\
& a_{\mathrm{F}}^{h}\left(\vartheta_{\mathrm{F}, h}^{n+1, i+1}, v_{h}\right)=\left\langle Q^{n+\theta}, v_{h}\right\rangle_{\Omega}+\left\langle q_{\mathrm{N}}^{n+\theta}, v_{h}\right\rangle_{\Gamma_{\mathrm{N}}^{\vartheta}}+\left\langle\alpha\left(\vartheta_{\mathrm{F}, h}^{n+1, i+1}-\vartheta_{\mathrm{S}, h}^{n+1, k}\right), v_{h}\right\rangle_{\Gamma_{\mathrm{SF}}}
\end{aligned}
$$

where $v_{h} \in V_{\mathrm{S}, h}^{0}$ in (11) and in $v_{h} \in V_{\mathrm{F}, h}^{0}$ in (12). Again we can take $k=i+1$ or $k=i$.

Apart from the fact that the physical models represented by Systems (9)-(10) and (11)-(12) are different, some conceptual differences have to be remarked. First, it is observed that the imposition of the transmission conditions is 'symmetric' for the fluid and the solid, contrary to the DirichletNeumann conditions in (9)-(10). Secondly, (11)-(12) does not require the calculation of the normal heat fluxes from the solid to the fluid, as needed in (10). This calculation is always involved in a finite element code, particularly for non-matching meshes between the fluid and the solid (see the following section). Finally, in the limit $\alpha \rightarrow \infty$ it can be shown that the solution of System (11)-(12) converges to the solution of System (9)-(10), the convergence rate being $\alpha^{-1}$. This can be proved using the analysis developed in [12]. Nevertheless, in our approach $\alpha$ has a physical meaning and, moreover, taking $\alpha$ large leads to ill-conditioning problems. 


\section{IMPLEMENTATION ASPECTS}

\subsection{A master-slave algorithm}

One important point of the iteration-by-subdomain strategy proposed is that we already had programs that solve the fluid dynamics problem and the structural problem. Then a master/slave algorithm was implemented by developing a third code (the master code) in order to control the iterative process. The MPICH2 library, an implementation of the MPI-2 standard, provides functions for process communications that are used to interchange the data needed to apply boundary conditions on each dedicated (slave) code. Some minor modifications on these codes are needed in order to exchange data with the master. In order to perform a calculation, input data for each subproblem needs to be generated and the master code starts the calculation by starting the slave process (this is only possible under MPI-2 standard). During the calculation, the master code needs to define the boundary conditions to be applied on each subproblem. The situation is illustrated in Figure 3.

\subsection{Boundary data interpolation}

Another aspect of the implementation that deserves a comment is the interpolation of the boundary conditions to be applied in one subdomain from the results obtained in the other subdomain. For each interface node, this interpolation is performed to find, in the mesh of the other subdomain, the element in which it is located, the so-called host element. The process is illustrated in Figure 4.

The element search strategy used in this study [9] is based on a quad-tree (oct-tree in three dimensions) algorithm. It consists of two steps: the preprocess in which a tree-like structure is built and a process in which the search is performed. In the preprocess, the host computational domain is embedded in a box taking the maximum and minimum nodes coordinates to define its coordinates. This box is then subdivided recursively into four boxes (eight boxes in three dimensions) until each box contains a prescribed (small) number of elements. Once this preprocess has been performed, the process to search the host element of a given point is faster. Given the test point coordinates

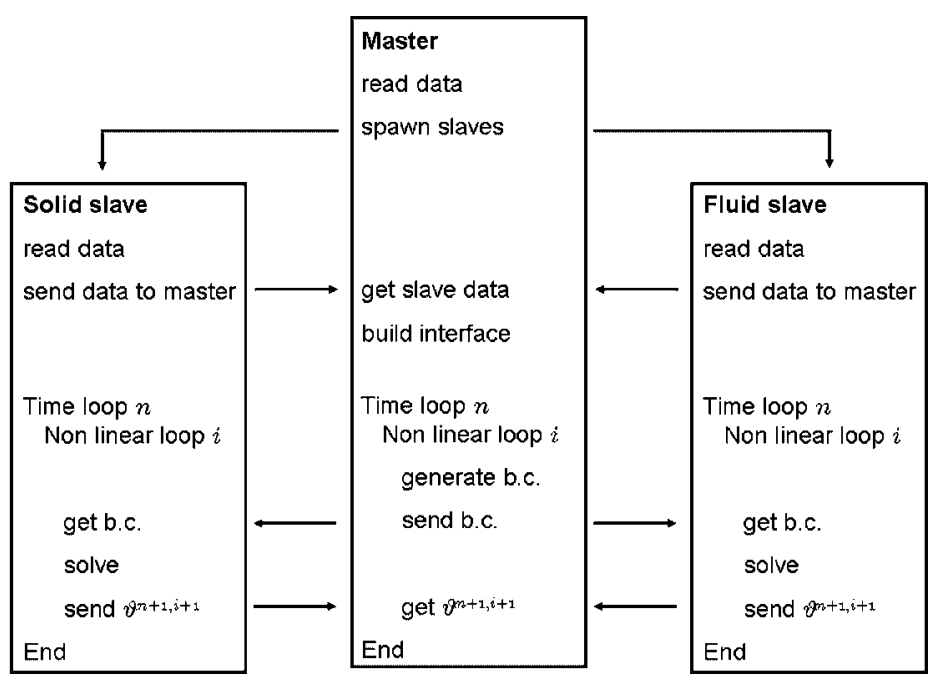

Figure 3. Master-slave implementation. 


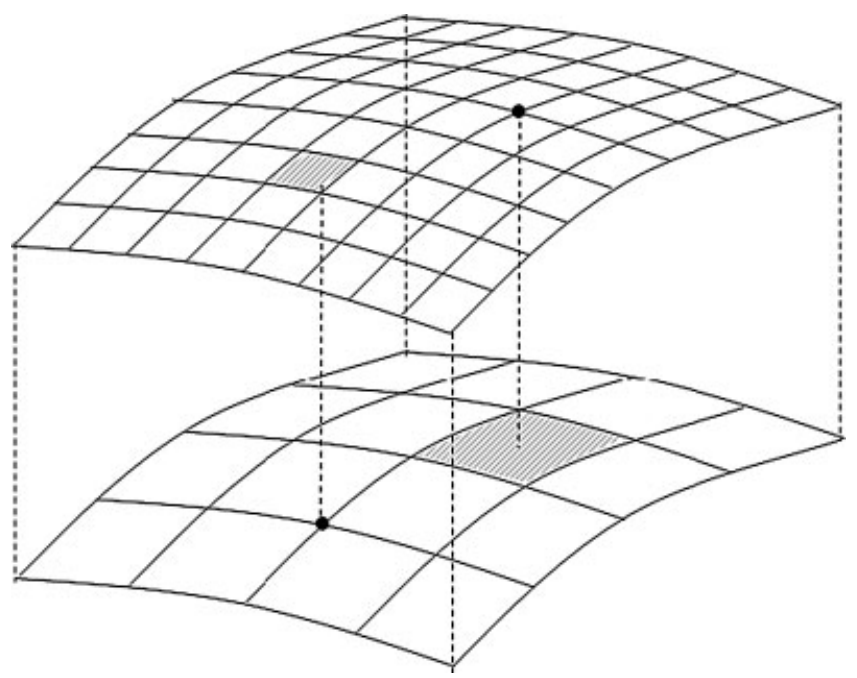

Figure 4. Boundary data interpolation.

$\mathbf{x}$ we recursively locate the boxes it belongs to and we find a small number of elements in which the point must be. Then on each element we perform a local coordinates test. If the coordinates on the parent domain of the standard isoparametric mapping are denoted by $\xi$, we have

$$
\mathbf{x}=\sum_{a} N^{a}(\boldsymbol{\xi}) \mathbf{x}^{a}
$$

and starting with $\mathbf{x}$ we solve this equation for $\xi$ using a Newton-Raphson procedure. The solution permits us to determine whether the point belongs to the element and whether it is the case we already have the shape functions on the host mesh evaluated at that point. They are then used to interpolate the needed boundary data.

\section{NUMERICAL EXAMPLES}

In this section we present two numerical examples. The first one is a very simple one-dimensional example intended to show the role played by the wall function approach when very thin boundary layers are created. The second example is a practical application of the thermal coupling described in this article.

\subsection{A one-dimensional example}

Assume that we have two different materials $\mathrm{F}$ and $\mathrm{S}$ on domains $\Omega_{\mathrm{F}}=[-1,0]$ and $\Omega_{\mathrm{S}}=[0,1]$, with conductivities $k_{\mathrm{F}}$ and $k_{\mathrm{S}}$, respectively, defined by

$$
\begin{aligned}
& k_{\mathrm{F}}=C \frac{1-\mathrm{e}^{-\gamma}}{1-\mathrm{e}^{-\gamma}+\gamma \mathrm{e}^{\gamma x}} \\
& k_{\mathrm{S}}=C \frac{1-\mathrm{e}^{-\gamma}}{1-\mathrm{e}^{-\gamma}+\gamma \mathrm{e}^{-\gamma x}}
\end{aligned}
$$



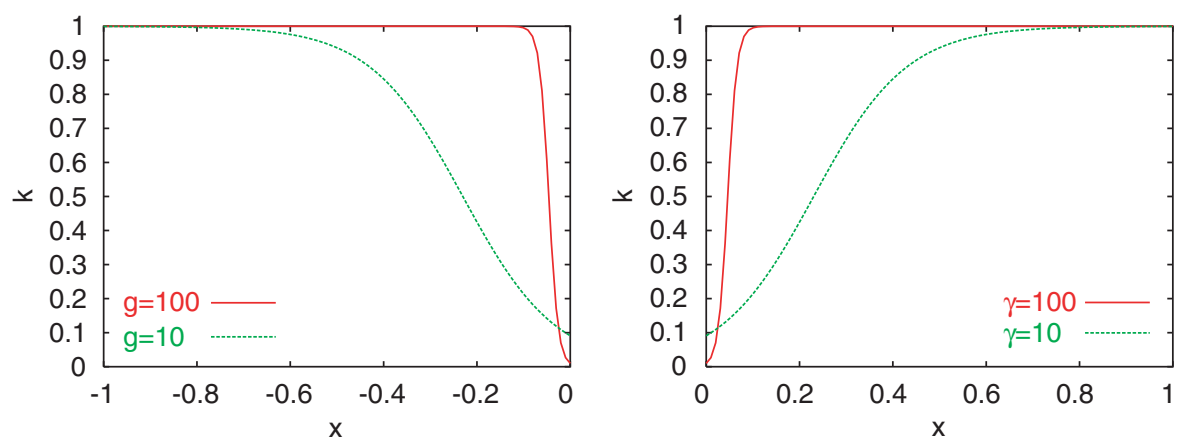

Figure 5. Thermal conductivity.

where $C$ and $\gamma$ are constants. Both coefficients have a boundary layer near $x=0$ and the constant $\gamma$ is a measure of the boundary layer width. The coefficients are shown in Figure 5 for $\gamma=10$ and $\gamma=100$ and $C=1$.

The problem can be expressed as

$$
\begin{aligned}
& -\frac{\mathrm{d}}{\mathrm{d} x}\left(k_{\mathrm{F}} \frac{\mathrm{d} \vartheta}{\mathrm{d} x}\right)=Q_{\mathrm{F}} \quad \text { in } \Omega_{\mathrm{F}} \\
& -\frac{\mathrm{d}}{\mathrm{d} x}\left(k_{\mathrm{S}} \frac{\mathrm{d} \vartheta}{\mathrm{d} x}\right)=Q_{\mathrm{S}} \quad \text { in } \Omega_{\mathrm{S}}
\end{aligned}
$$

with the transmission conditions

$$
\begin{gathered}
-k_{\mathrm{F}} \frac{\mathrm{d} \vartheta}{\mathrm{d} x}=-k_{\mathrm{S}} \frac{\mathrm{d} \vartheta}{\mathrm{d} x} \quad \text { at } x=0 \\
\vartheta_{\mathrm{F}}(0)=\vartheta_{\mathrm{S}}(0)
\end{gathered}
$$

and the boundary conditions

$$
\begin{gathered}
\vartheta_{\mathrm{F}}(-1)=1 \\
\vartheta_{\mathrm{S}}(1)=0
\end{gathered}
$$

The exact solution to this problem is

$$
\begin{aligned}
& \vartheta_{\mathrm{F}}(x)=\frac{1}{2}\left(-x+\frac{1-\mathrm{e}^{\gamma x}}{1-\mathrm{e}^{-\gamma}}\right) \\
& \vartheta_{\mathrm{S}}(x)=\frac{1}{2}\left(-x-\frac{1-\mathrm{e}^{-\gamma x}}{1-\mathrm{e}^{-\gamma}}\right)
\end{aligned}
$$

We have solved this problem in the case of $\gamma=100$ using the first domain decomposition strategy using three different meshes of 10,20 and 40 elements. The solution is compared with the analytical solution in Figure 6. We have also solved this problem using the second approach using a mesh of 10 elements and the result is compared with the one obtained by the previous method and with the analytical solution in Figure 7. We note that using the first strategy, a better solution can be 

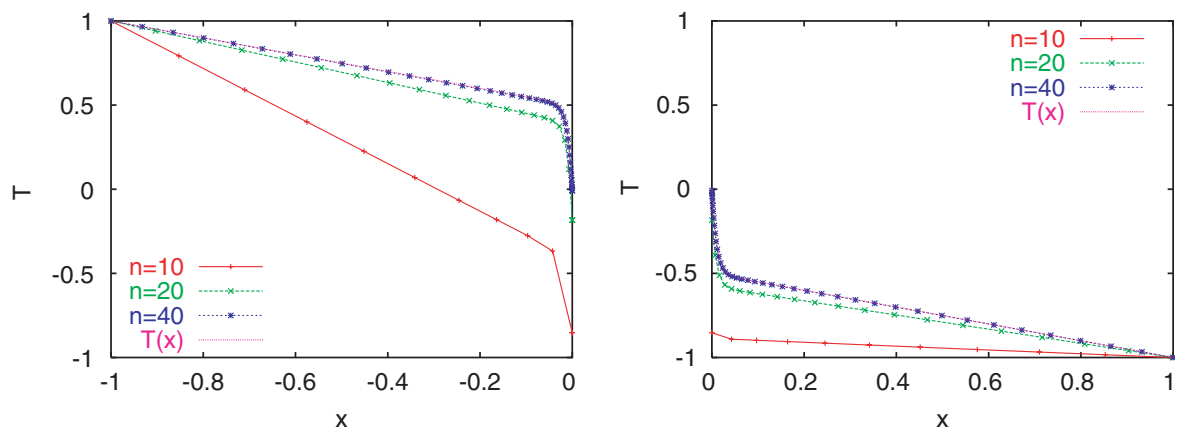

Figure 6. Finite element solution obtained using domain decomposition with two subdomains and meshes of 10, 20 and 40 elements compared with the analytic solution.
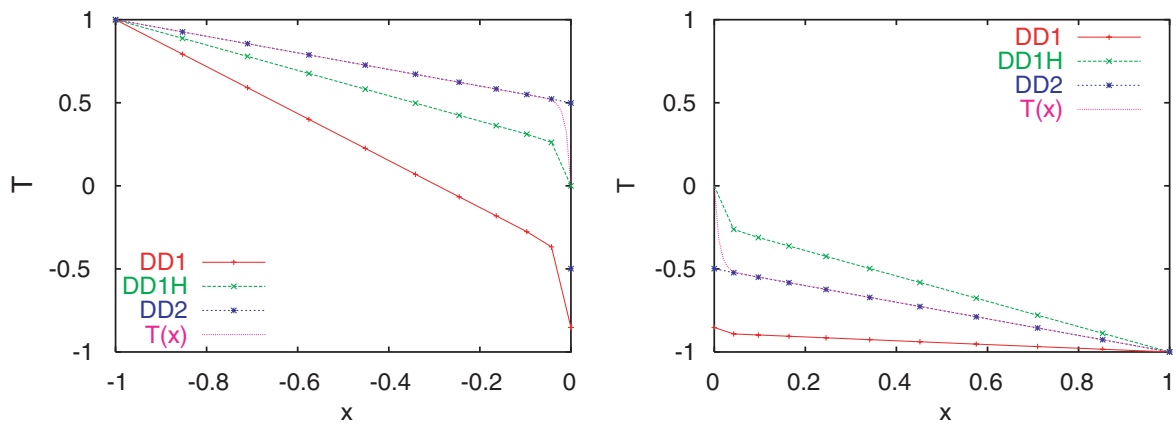

Figure 7. Finite element solution obtained using domain decomposition with two subdomains (DD1), with two subdomains and second-order interpolation of the normal fluxes (DD1H) and with three subdomains (DD2) compared with the analytical solution.

obtained if a second-order approximation of the normal derivatives is made, which implies the need of building an appropriate extrapolation as shown also in Figure 7. This is not a simple task in general domains with unstructured grids.

In any case, it is clear that the second method gives much better results in the case of a coarse discretization. The accuracy of this approach depends on the choice of the coefficient $\alpha$. The optimal value used here is found noting that, when $\gamma \rightarrow \infty$, the exact solution tends to

$$
\begin{aligned}
& \vartheta_{\mathrm{F}}(x)=-\frac{1}{2} x+\frac{1}{2} \\
& \vartheta_{\mathrm{S}}(x)=-\frac{1}{2} x-\frac{1}{2}
\end{aligned}
$$

and the conduction coefficients tend to 1 (except at $x=0$ where both are 0 ) from where we obtain $\alpha=\frac{1}{2}$.

\subsection{A fire in a tunnel}

A fire is a complex phenomenon whose detailed simulation involves many different aspects that we are not considering in this study. Here we have used a simple model that considers the fire as 

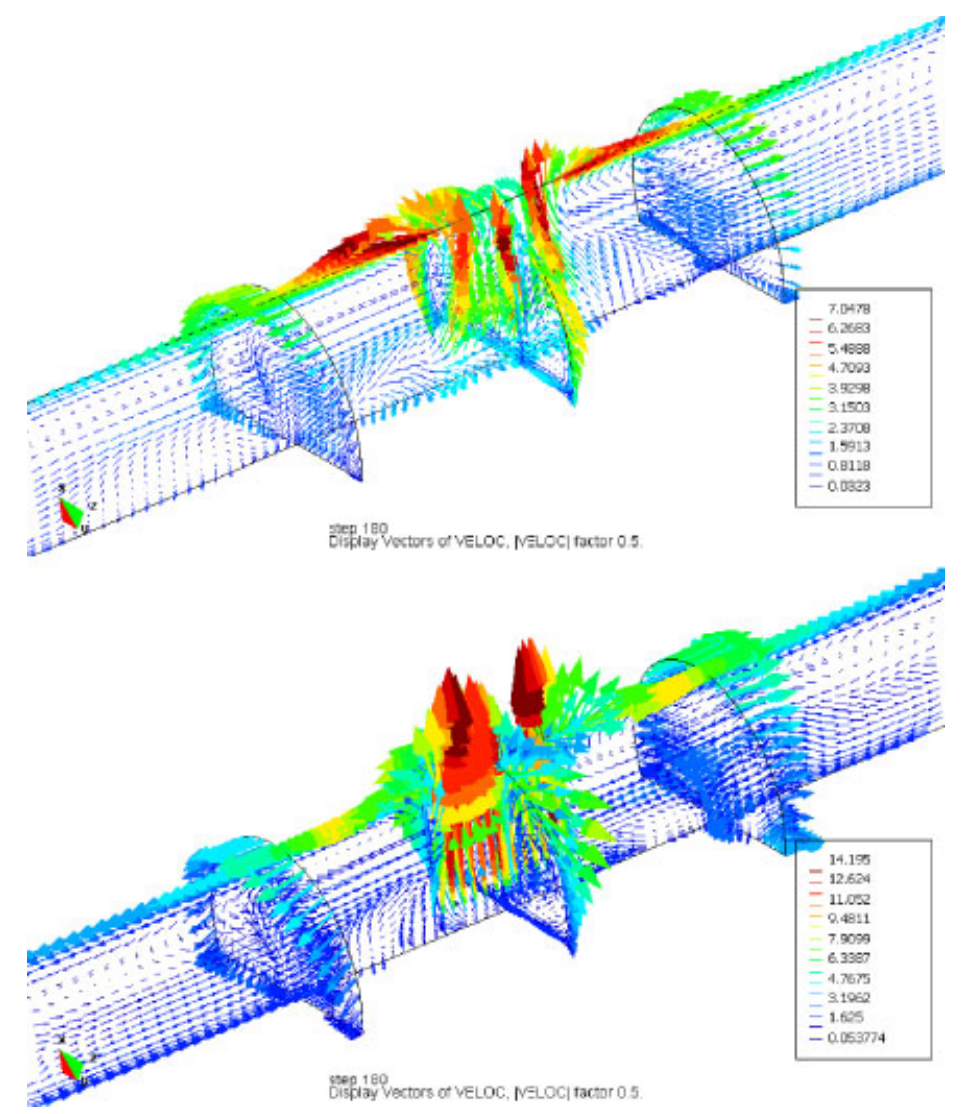

Figure 8. Velocity field at $t=180 \mathrm{~s}$ for $Q=1.25 \mathrm{MW} / \mathrm{m}^{3}$ (top) and $Q=4.0 \mathrm{MW} / \mathrm{m}^{3}$ (bottom).

a source of heat, without taking into account the exact reactive mechanism, as this would imply a precise knowledge of the chemical components of the fuel. The heat released during a fire, which is between 1 and $100 \mathrm{MW}$, is partially dissipated by the flow and partially transported toward the concrete structure where it is finally dissipated. Thus, the heat transfer involves both the behavior of the fluid inside the tunnel and the structural behavior of the concrete and it is therefore necessary to solve a coupled problem.

We solve the problem using the low Mach number approximation to the compressible flow equations. This model takes into account the compressibility of the fluid but removes the acoustic modes [13]. Unlike the Boussinesq approximation, strong temperature and density gradients are allowed. The numerical treatment of the low Mach number equations is described in [14].

The high Reynolds number of the problem implies the need of taking turbulence into account. We do this by introducing a Smagorinsky eddy [15], which is defined as

$$
\mu^{\mathrm{t}}=\rho c_{s} \Delta^{2}\left[\boldsymbol{\varepsilon}^{\prime}(\mathbf{u}): \boldsymbol{\varepsilon}^{\prime}(\mathbf{u})\right]^{1 / 2}
$$

where $c_{s}$ is an empirical constant, $\Delta$ a characteristic length usually taken as the mesh size and $\boldsymbol{\varepsilon}^{\prime}(\mathbf{u})$ is the deviatoric part of the rate of deformation tensor. A subgrid thermal conductivity is 

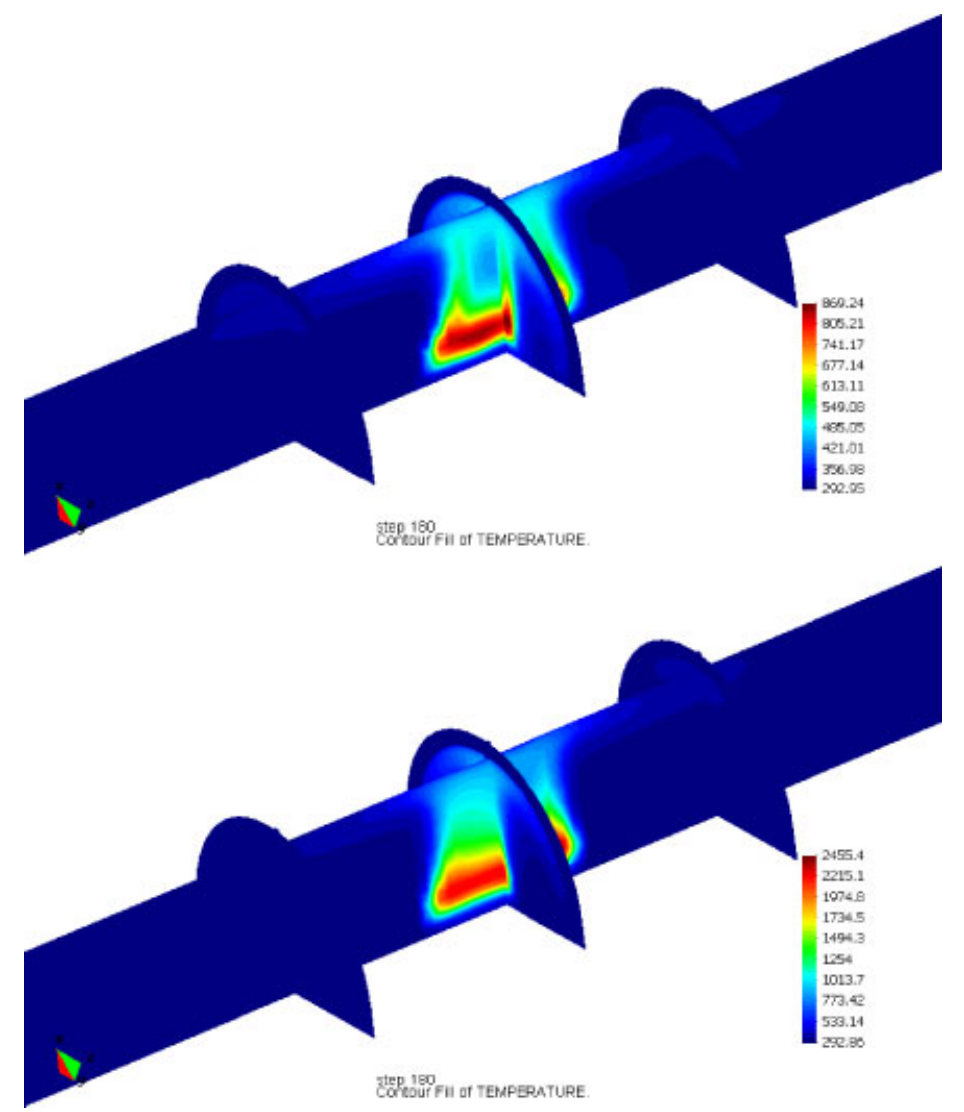

Figure 9. Temperature field at $t=180 \mathrm{~s}$ for $Q=1.25 \mathrm{MW} / \mathrm{m}^{3}$ (top) and $Q=4.0 \mathrm{MW} / \mathrm{m}^{3}$ (bottom).

also added. It is defined in terms of the subgrid viscosity as

$$
k^{\mathrm{t}}=\frac{\mu^{\mathrm{t}} c_{p}}{P r^{\mathrm{t}}}
$$

where $P r^{\mathrm{t}}$ is the turbulent Prandtl number, which is assumed to be constant (and taken to be 0.5).

Two simulations were carried out considering heat sources of 10 and $30 \mathrm{MW}$, which correspond to a small size fire (a car, for example) distributed in a volume of $8 \mathrm{~m}^{3}$. Based on experimental results, a typical wind in a tunnel in the absence of fire has a velocity of about $0.5 \mathrm{~m} / \mathrm{s}$. A preliminary calculation was performed to reproduce the initial state of a wind flowing through the tunnel, which was obtained applying a pressure difference between the tunnel inlet and outlet. On the tunnel walls Neumann boundary conditions based on universal profiles were applied (wall laws). Boundary conditions for temperature were defined to reproduce the real situation as close as possible. On the tunnel walls a Robin-type condition as in (11)-(12) was applied using a convection coefficient suggested by laboratory experiments and the temperature on the concrete walls was fixed. On the entrance and exit of the tunnel, Neumann boundary conditions were considered. 

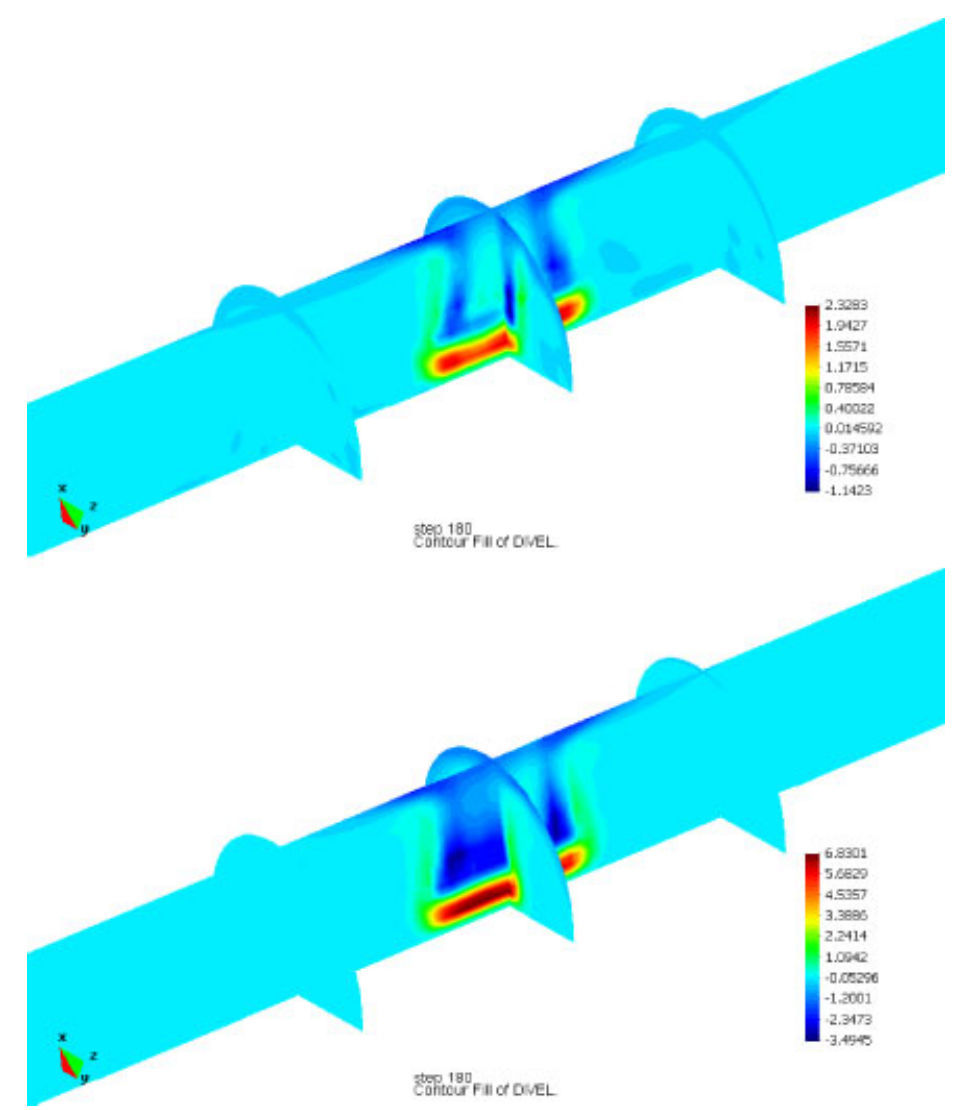

Figure 10. Divergence of the velocity field at $t=180 \mathrm{~s}$ for $Q=1.25 \mathrm{MW} / \mathrm{m}^{3}$ (top) and $Q=4.0 \mathrm{MW} / \mathrm{m}^{3}$ (bottom).

The physics of the flow is quite complex and the temporal evolution is chaotic. When the heating starts, strong buoyancy forces determine the formation of a plume and recirculation zones that now, in contrast to the previous example, are fully tridimensional and of complex structure. In Figure 8 the velocity field at 3 min after the starting of the heating is shown and in Figure 9 the corresponding temperature field is shown. Both figures show a detail of the fire zone introducing cutting planes that intersect the fire zone. The heat source generates the plume that can be clearly observed in Figure 8, where an expansion of the flow is also apparent. This expansion is better shown in Figure 10, where contour lines of divergence of the velocity are shown. They have been obtained by projecting velocity gradients on the finite element space.

In both calculations we used a time step $\delta t=1 \mathrm{~s}$. The nonlinear equations describing the flow are solved using two nested loops, an external global loop and internal loops for the momentum equations and for the temperature equation (which is non-linear in the low Mach number case because of the dependence of the density on the temperature). The external loop is also used to account for the domain decomposition coupling. A maximum number of 5 iterations in the external loop were performed with a convergence tolerance of $10^{-3}$ for the velocity and of $10^{-4}$ for the 
temperature. In most steps, 3 iterations were enough to achieve convergence and only in few steps the temperature residual after 5 iterations was around $0.2 \times 10^{-3}$ (the velocity residual was always under the prescribed tolerance). The linear system has been solved using a GMRES [16] preconditioned using an ILUT strategy described in [17]. As pointed out in [14], this technique is effective when large time steps are used.

\section{CONCLUSIONS}

In this article, we have described different aspects related to the numerical approximation of the thermal coupling between a fluid and a solid. Our basic strategy has been to pose the problem in a domain decomposition framework. This has allowed us to propose two alternatives to treat the interface coupling, namely, a classical one considering a perfect thermal contact (continuity of temperatures and heat flux) and another one based on the use of wall functions, which leads to a heat flux proportional to the temperature jump between the fluid and the solid. This surfaceconvection-like transmission condition depends on a coefficient to which we have given a new expression in terms of the parameters of the wall function approach. When this coefficient increases the perfect thermal contact condition is recovered.

We have also discussed the iteration-by-subdomain strategy we have implemented using a master-slave strategy. Again, the domain decomposition framework turns out to be crucial to formulate this (otherwise standard) iterative strategy.

From the practical point of view, we have found that the algorithmic framework presented here is very handful, easy to implement once the basic dedicated codes are available and, what is more important, robust (in accordance with results known from the literature). An application example of the overall formulation has been presented.

\section{REFERENCES}

1. Incropera F, Dewitt D. Fundamentals of Heat and Mass Transfer (4th edn). Wiley: New York, 1996.

2. Gawin D, Pesavento F, Schrefler BA. Simulation of damage-permeability coupling in hygro-thermo-mechanical analysis of concrete at high temperature. Communications in Numerical Methods in Engineering 2002; 18: 113-119.

3. Jayatilleke CLV. The influence of Prandtl number and surface roughness on the resistance of laminar sub-layer to momentum and heat transfer. Progress in Heat Transfer, vol. 1. Pergamon Press: Oxford, 1969.

4. Codina R, Houzeaux G. Numerical approximation of the heat transfer between domains separated by thin walls. International Journal for Numerical Methods in Fluids 2006; 52:963-986.

5. Codina R. Stabilized finite element approximation of transient incompressible flows using orthogonal subscales. Computer Methods in Applied Mechanics and Engineering 2002; 191(39-40):4295-4321.

6. Codina R, Principe J. Dynamic subscales in the finite element approximation of thermally coupled incompressible flows. International Journal for Numerical Methods in Fluids 2007; 54(6-8):707-730.

7. Codina R, Principe J, Guasch O, Badia S. Time dependent subscales in the stabilized finite element approximation of incompressible flow problems. Computer Methods in Applied Mechanics and Engineering 2007; 196(21-24): 2413-2430.

8. Hughes TJR. Multiscale phenomena, Green's functions, the Dirichlet-to-Neuman formulation, subgrid scale models, bubbles and the origins of stabilized methods. Computer Methods in Applied Mechanics and Engineering 1995; 127:387-401.

9. Houzeaux G. A geometrical domain decomposition method in computational fluid dynamics. Ph.D. Thesis, Escola Tècnica Superior d'Enginyers de Camins, Canals i Ports, Universitat Politècnica de Catalunya, Barcelona, 2002.

10. Giles M. Stability analysis of numerical interface conditions in fluid-structure thermal analysis. International Journal for Numerical Methods in Fluids 1997; 25:421-436. 
11. Roe B, Haselbacher A, Geubelle P. Stability of fluid-structure thermal simulations on moving grids. International Journal for Numerical Methods in Fluids 2007; 54:1097-1117.

12. Chacón Rebollo T, Chacón Vera E. Study of a non-overlapping domain decomposition method: Poisson and Stokes problems. Applied Numerical Mathematics 2004; 48:169-194.

13. Principe J, Codina R. On the low Mach number and the Boussinesq approximations for low speed flows. Technical Report, CIMNE, Publication PI-313, 2008.

14. Principe J, Codina R. A stabilized finite element approximation of low speed thermally coupled flows. International Journal for Numerical Methods in Heat and Fluid Flow, to appear.

15. Sagaut P. Large Eddy Simulation for Incompressible Flows. Scientific Computing. Springer: Berlin, 2001.

16. Saad Y. Iterative Methods for Sparse Linear Systems (1st edn). PWS Publishing Company: Boston, 1996.

17. Saad Y. Ilut: a dual threshold incomplete ILU factorization. Numerical Linear Algebra with Applications 1994; 1:387-402. 